8-15-1999

\title{
Electrical Transport, Magnetism, and Spin-state Configurations of High-Pressure Phases of FeS
}

Takele Seda

Western Washington University, takele.seda@wwu.edu

G. R. Hearne

Follow this and additional works at: https://cedar.wwu.edu/physicsastronomy_facpubs

Part of the Physics Commons

\section{Recommended Citation}

S. Takele and G. R. Hearne: Electrical transport, magnetism, and spin-state configurations of high-pressure phases of FeS, Phys. Rev. B 60(1999) 4401.

This Article is brought to you for free and open access by the College of Science and Engineering at Western CEDAR. It has been accepted for inclusion in Physics \& Astronomy by an authorized administrator of Western CEDAR. For more information, please contact westerncedar@wwu.edu. 


\title{
Electrical transport, magnetism, and spin-state configurations of high-pressure phases of FeS
}

\author{
S. Takele and G. R. Hearne \\ Department of Physics, University of the Witwatersrand, Private Bag 3, P.O. Wits 2050, Johannesburg-Gauteng, South Africa
}

(Received 16 February 1999)

\begin{abstract}
Both the nature of the electrical transport and magnetism of high-pressure phases of FeS have been elucidated in relation to the spin-state configuration of Fe. This has been achieved by combined ${ }^{57} \mathrm{Fe}$ Mössbauer spectroscopy and resistance measurements on samples pressurized in miniature gem-anvil pressure cells to $\sim 12 \mathrm{GPa}$ in the range $300-5 \mathrm{~K}$. Hexagonal $\mathrm{FeS}$ in the range 3-7 GPa exhibits magnetic nonmetallic behavior, whereas monoclinic FeS beyond $\sim 7 \mathrm{GPa}$ is diamagnetic and nonmetallic. This is compelling experimental evidence to show that, along the room-temperature isotherm, hexagonal FeS has thermally activated charge carriers and a high-spin magnetic-electronic configuration, whereas monoclinic FeS adopts a magnetically quenched low-spin state and concomitant filled valence band. [S0163-1829(99)01633-1]
\end{abstract}

FeS is of interest to both condensed matter physics and planetary science. It is an antiferromagnetic semiconductor ${ }^{1,2}$ under ambient conditions and is therefore considered to be a strongly correlated electron system, ${ }^{3}$ permitting an interesting comparison to be made of such narrow $3 d$-band phenomena in transition-metal sulphides against those in transitionmetal oxides. It is also a likely component of the cores of terrestrial planets and of meteorites.

A variety of crystallographic structures adopted by troilite FeS spanning ambient to combined high pressuretemperature $(P-T)$ conditions of from $20 \mathrm{GPa}$ to $1000 \mathrm{~K}$ has been comprehensively investigated in previous structural studies, yet the magnetic and electrical properties of the high $P-T$ phases have been less extensively investigated. The goal of this work is to elucidate the magnetic-electronic properties of the high-pressure phases, and this has been achieved by separate ${ }^{57} \mathrm{Fe}$ Mössbauer and electrical-transport measurements on FeS samples pressurized in gem-anvil cells.

The $P-T$ structural phase diagram of FeS from the most recent $\mathrm{X}$-ray pressure studies by Kusaba $\mathrm{et} \mathrm{al} .{ }^{4}$ using a largevolume press is shown in Fig. 1. The compound is seen to exhibit a number of structural transitions under various $P-T$ conditions. Under ambient conditions troilite $\mathrm{FeS}$ is a hexagonal superstructure derivative of the NiAs unit cell and has axis lengths $a=\sqrt{3} c_{f}$ and $c=2 c_{f}$, where $a_{f}$ and $c_{f}$ are the lattice parameters of a fundamental $\mathrm{NiAs}$ subcell. The lattice parameters of the troilite unit cell depicted in Fig. 1 are typically about $a=5.966 \AA$ and $c=11.76 \AA$, and there is a phase transformation to the NiAs-type structure of unit-cell dimensions $\left(a_{f}, c_{f}\right)$ at ambient pressure and an onset temperature of $\sim 413 \mathrm{~K}^{4}$ Increasing the pressure at room temperature ${ }^{4,5}$ results in a structural transformation at $\sim 3.5 \mathrm{GPa}$ to a hexagonal NiAs-type superstructure having unit-cell dimensions $\left(2 a_{f}, c_{f}\right)$. Further increase in pressure along the roomtemperature isotherm results in another structural transformation at $\sim 7 \mathrm{GPa}$ to a new phase that may be indexed by a monoclinic unit cell in the space group $P 2_{1}$ or $P 2_{1} / \mathrm{m}$, although details of the new structure (e.g., atomic positions) are perhaps less well established. ${ }^{5,6}$

Previous attempts have been made to elucidate the magnetism and nature of electrical transport of the high- $P$ phases occurring beyond $\sim 3.5$ and $\sim 7 \mathrm{GPa}$, but there appears to be ambiguity and speculation in this regard because both the ${ }^{57} \mathrm{Fe}$ Mössbauer-magnetic ${ }^{7,8}$ and electrical-transport ${ }^{4,9,10}$ characterization have been limited to studies in the vicinity of room temperature.

A precipitous drop in the room-temperature resistance at $\sim 3 \mathrm{GPa}$ in previous electrical-transport studies ${ }^{4,10}$ has been ascribed to a semiconductor-metal transition. However, the change in resistance at $\sim 3 \mathrm{GPa}$ is not a conclusive feature of metallization and may be a consequence of a transformation to a new structural phase having a much smaller band gap. Reliable conclusions are perhaps best drawn after establishing the temperature coefficient of resistance, $d R / d T$.

The pressure-induced disappearance of a magnetic signature in the ${ }^{57} \mathrm{Fe}$ Mössbauer spectrum at $\sim 7 \mathrm{GPa}$ and room temperature $\mathrm{e}^{7,8}$ has been attributed to a collapsed magnetic moment, either due to electron delocalization, suggesting a metallic high- $P$ phase, ${ }^{11}$ or due to spin crossover of the $\mathrm{Fe}$ atomic spin $S$ from the original high spin $S=2$ $\left[\mathrm{Fe}^{2+}(3 d): t_{2 g}^{4} e_{g}^{2}\right]$ to diamagnetic low spin $S=0$ $\left[\mathrm{Fe}^{2+}(3 d): t_{2 g}^{6} e_{g}^{0}\right]$. A drastic change in the magnetic ordering

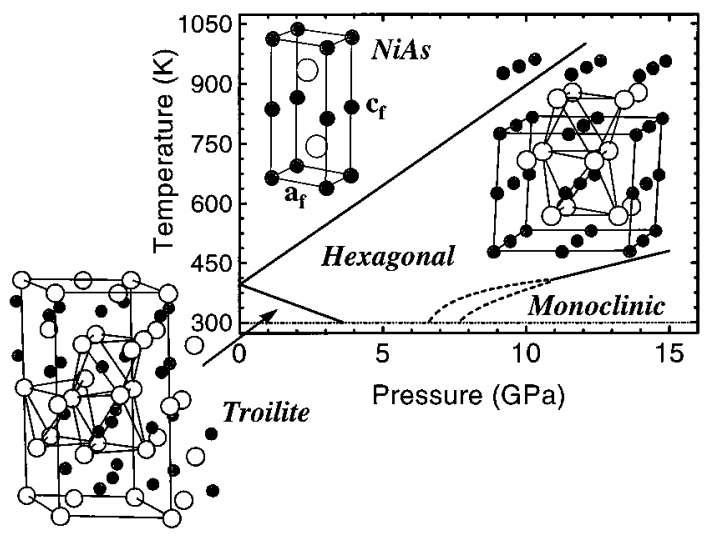

FIG. 1. Structural phase diagram of FeS derived from recent x-ray studies of Kusaba et al. under various $P-T$ conditions (Refs. 4 and 6). The dashed phase boundaries (6.5-7.5 GPa) demarcate a range where two phases coexist. Straight solid lines in the structures show the unit-cell as well as the octahedral coordination of $\mathrm{Fe}$ (small shaded circles) by S (larger open circles) atoms. 
temperature to below room temperature also has to be considered as a possibility due to changes in interatomic distances and bond angles associated with a $\sim 5 \%$ change in unit-cell volume at the hexagonal $\rightarrow$ monoclinic transition. ${ }^{4}$

A temperature-dependent study of both ${ }^{57} \mathrm{Fe}$ Mössbauer and electrical-resistance data to practically accessible liquidhelium temperatures may help to eliminate any ambiguity regarding both the magnetic-electronic state of $\mathrm{Fe}$ and the nature of the electrical transport of the high- $P$ phases. We have therefore executed variable-temperature studies on polycrystalline powdered $\mathrm{FeS}$ samples at pressures up to $\sim 12 \mathrm{GPa}$ by means of both ${ }^{57} \mathrm{Fe}$ Mössbauer spectroscopy in a diamond-anvil cell (300-5 K) (Ref. 12) and electricalresistance measurements in a gem-anvil cell $(300-80 \mathrm{~K})$. A sample of troilite FeS enriched to $\sim 25 \%{ }^{57} \mathrm{Fe}$ has been synthesized in milligram quantities by reacting the elements in an evacuated quartz tube at $700^{\circ} \mathrm{C}$ for a few days. Mössbauer spectra under ambient conditions yield almost identical hyperfine interaction parameters to that obtained by Kobayashi et $\mathrm{al}^{8}$ for a sample synthesized using natural abundance materials and which they deduced to have a chemical formula of $\mathrm{Fe}_{0.996} \mathrm{~S}$. X-ray-diffraction studies independently confirm the stoichiometry of our material as being close to $\mathrm{Fe}_{0.996} \mathrm{~S}$. In our resistance-pressure experiments, four-probe current-reversing resistance-temperature $(R-T)$ measurements have been performed with a dipstick-type arrangement in a storage Dewar using cubic-zirconia gems as pressure anvils, fine gold-wire electrodes, and an insulating mica gasket to constitute the confining sample cavity. ${ }^{13}$ The luminescence line shift at room temperature from fine ruby grains loaded into the sample cavity has been used to determine the pressure.

Figures 2(a)-2(c) illustrate selected ${ }^{57} \mathrm{Fe}$ Mössbauer spectra measured at room temperature in each of the three phases occurring along the room-temperature isotherm as proposed in the recent structural study of Kusaba et al. ${ }^{4}$ This is in agreement with the most recent Mössbauer pressure data of Kobayashi et al., ${ }^{8}$ showing a collapse of the magnetic sextet at room temperature to yield only a quadrupole-interaction doublet. Our Mössbauer pressure studies to cryogenic temperatures [e.g., Fig. 2(d)] show that the nonmagnetic quadrupole doublet persists to the lowest attainable temperature of $5 \mathrm{~K}$, strongly suggestive of diamagnetic behavior. The possibility that the monoclinic phase has a spin-ordering temperature below $300 \mathrm{~K}$ at pressures beyond $\sim 7 \mathrm{GPa}$ may therefore be discounted.

Normalized resistance-temperature measurements at selected pressures spanning the different structural phases upon rising pressure have been depicted in Fig. 2(e), demonstrating that a negative temperature coefficient of resistance, $d R / d T<0$, obtains in the hexagonal and monoclinic structures of Fig. 1. This is indicative of behavior typical of a small-band-gap semiconductor, in contradiction to the speculation of all previous work restricted to measurements at or close to room temperature that claim intermediate- $P(3.5-7$ $\mathrm{GPa})$ and high- $P(>7 \mathrm{GPa})$ phases to be metallic., ${ }^{4,8}$ Arrhenius-type plots and analysis of the data in Fig. 2(e) show (semiconducting) carrier transport in the high-pressure phases near room temperature to involve activation energies comparable to that of troilite $\mathrm{FeS}$ at ambient pressure, namely, $10-20 \mathrm{meV} .^{2}$
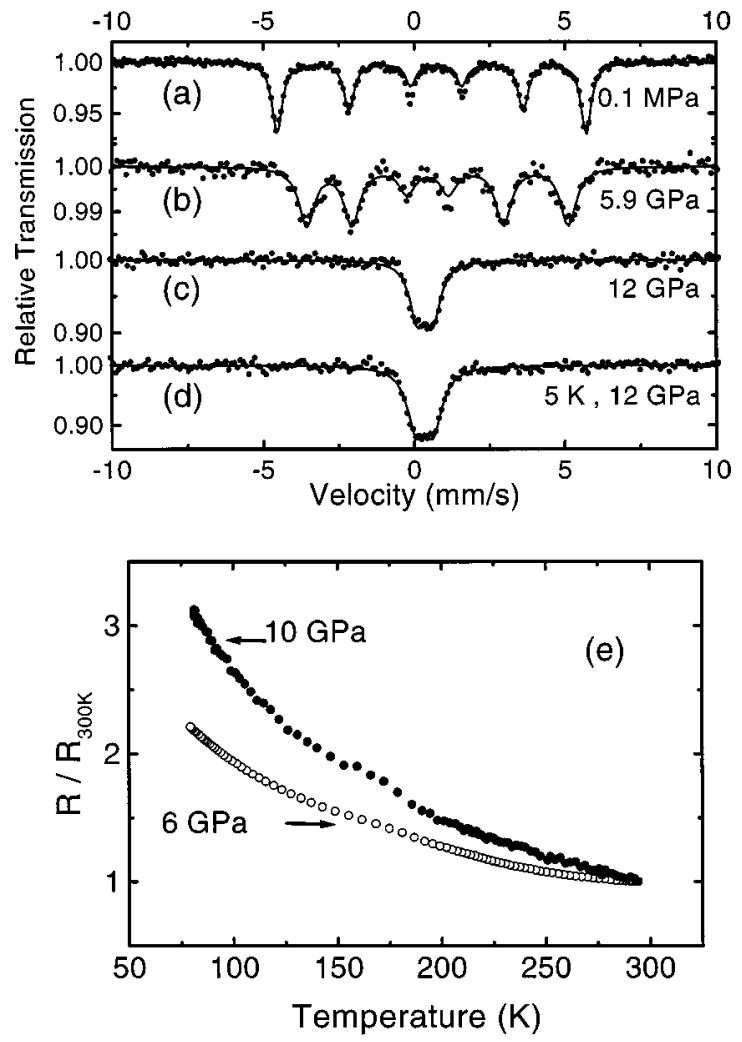

FIG. 2. ${ }^{57} \mathrm{Fe}$ Mössbauer spectra of FeS in the various structural phases at elevated pressures (a)-(c). Magnetic spectra at pressures in (a) and (b) have been recorded at room temperature, whereas the spectra at higher pressure in (c) and (d) indicate nonmagnetic behavior at both room temperature and $5 \mathrm{~K}$, respectively. Solid lines are theoretical fits to the data. The bottom panel (e) has resistancetemperature data at selected pressures normalized to the resistance at room temperature.

The magnetic and electrical properties of transition-metal compounds may be primarily influenced by the electronic configuration of the cation. In octahedral coordination this depends on the relative values of interatomic $(\mathrm{Fe}-\mathrm{S})$ and intra-atomic ( $\mathrm{Fe}: 3 d$ spin pairing) exchange interaction energies $\Delta_{\text {cf }}$ and $\Delta_{\text {sp }}$, respectively. ${ }^{14}$ The crystal-field splitting $\Delta_{\text {cf }}$ of $\sim 1-2 \mathrm{eV}$ represents, within a ligand-field picture, the energy difference between threefold-degenerate $3 d: t_{2 g}$ and doubly degenerate $3 d: e_{g}$ orbitals of the cation in the $\mathrm{FeS}_{6}$ octahedron; see Fig. 3(a). Alternatively, $\Delta_{\text {cf }}$ is a measure of the difference in strength of $\pi$-bond and $\sigma$-bond cation covalent mixing with ligand orbitals. Typical intra-atomicexchange stabilization energies $\Delta_{\mathrm{sp}} \sim 2-3 \mathrm{eV}$ favor maximal unpaired electron spins and associated orbital occupation to minimize interelectronic repulsion (Hund's rule). Upon increasing pressure and a corresponding reduction of the unitcell volume, $\Delta_{\text {cf }}$ may become comparable to or exceed $\Delta_{\mathrm{sp}}, \Delta_{\mathrm{cf}} \geqslant \Delta_{\mathrm{sp}}$. It is then energetically favorable for majorityspin $e_{g}(\uparrow)$-derived electrons to occupy $\pi$-bonding orbitals as $t_{2 g}(\downarrow)$-derived electrons [see evolution of Fig. 3(b) to Fig. 3 (c) ] representing the high-spin to low-spin spin-pairing transition that leads to a significant alteration of the electronic configuration.

The magnetic nonmetallic behavior of hexagonal $\mathrm{FeS}$ (3-7 GPa) suggests a high-spin $3 d: t_{2 g}^{4} e_{g}^{2}$ cation electronic configuration [see Fig. 3(b)] and thermally activated charge 


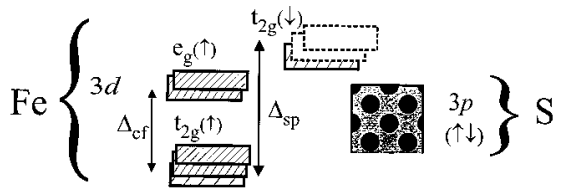

(a)

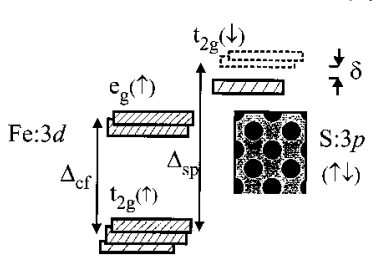

(b) $\Delta_{\mathrm{cf}}<\Delta_{\mathrm{sp}}$

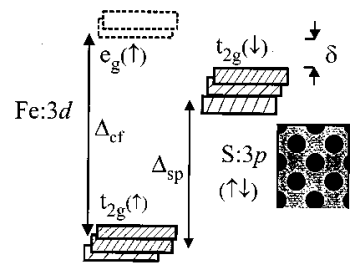

(c) $\Delta_{\mathrm{cf}}>\Delta_{\mathrm{sp}}$
FIG. 3. Schematic of the possible energy band structure of FeS for various electronic configurations. The highest occupied $\mathrm{Fe}: 3 d$ and $\mathrm{S}: 3 p$ bands are shown as solid rectangles, and symbols are defined in the text: (a) metallic high-spin state, (b) high-spin state with semiconducting gap $\delta$, and (c) semiconducting low-spin state. In the case of (b) and (c), the effect of on-site Coulomb repulsion, the Hubbard $U$, is implicitly included, possibly giving rise to the gap $\delta$. Shaded $3 d$ electronic states then represent lower Hubbard subbands.

carriers. The high-spin state has a partially filled minorityspin $t_{2 g}(\downarrow)$-derived subband contribution to the $3 d$ valence band, anticipated to render metallic behavior to FeS in conventional band theory [see Fig. 3(a)]. Therefore the nonmetallic behavior must be attributable to a gap $\delta$ in the $t_{2 g}(\downarrow)$ subband; see Fig. 3(b). The gap may originate either or both from strong electron correlations (on-site Coulomb repul- sion) represented by the Hubbard splitting $U$ of the $3 d$ band at narrow enough bandwidths ${ }^{15,16}$ or from pronounced $\mathrm{Fe}-\mathrm{Fe}$ interactions considered possible across shared faces of $\mathrm{FeS}_{6}$ octahedra along the $c$ axis ${ }^{17}$ in the structure of the hexagonal phase illustrated in Fig. 1.

The nonmetallic and diamagnetic state of the monoclinic phase at $P>7 \mathrm{GPa}$ is consistent with a fully occupied $t_{2 g}$-derived valence-band structure as a result of spin pairing to yield a low-spin $3 d: t_{2 g}^{6} e_{g}^{0}$ electronic configuration having quenched atomic spin $S=0$, depicted in Fig. 3(c). Kusaba et $a l .{ }^{4}$ have shown that from ambient pressure up to $\sim 7 \mathrm{GPa}$, preceding the structural transition to the monoclinic phase, there is a $\sim 10 \%$ reduction in unit-cell volume $V$ and an additional $\sim 5 \%$ reduction at the transition. Since $\Delta_{\mathrm{cf}}$ $\propto V^{-5 / 3}$, derived from the inverse fifth power dependence of $\Delta_{\text {cf }}$ on average Fe-S interatomic distance $R,{ }^{14}$ we anticipate that a $10 \%$ reduction in unit-cell volume alone for $\mathrm{FeS}$ would increase $\Delta_{\text {cf }}$ by $\sim 20 \%$ from an ambient estimate of $\sim 1.6 \mathrm{eV}$ (Ref. 14, p. 276) up to $\sim 1.9 \mathrm{eV}$ at $\sim 7 \mathrm{GPa}$. At this pressure, $\Delta_{\text {sp }}$ may be anticipated to decrease from its ambient estimate of 1.7-2.0 eV (Ref. 14, p. 29) due to increased cation-ligand orbital mixing. Thus the reduction in unit-cell volume up to "modest" pressures of $\sim 7 \mathrm{GPa}$ may quite plausibly lead to the spin-crossover condition $\Delta_{\mathrm{cf}} \geqslant \Delta_{\mathrm{sp}}$, invoking the electronic change depicted in the sequence Fig. 3(b) $\rightarrow$ Fig. 3(c).

Note added in proof. A detailed XRD pressure study has recently been performed to elucidate the phase occurring at $P>7 \mathrm{GPa}$ at room temperature. The study confirms that the structure is monoclinic (see Ref. 18).

Financial support from the NRF-ORP (Pretoria) is acknowledged with gratitude. L. M. Madzwara assisted in the initial stages of this project.
${ }^{1}$ J. L. Horwood, M. G. Townsend, and A. H. Webster, J. Solid State Chem. 17, 35 (1976).

${ }^{2}$ J. R. Gosselin, M. G. Townsend, and R. J. Tremblay, Solid State Commun. 19, 799 (1976).

${ }^{3}$ K. Shimada, T. Mizokawa, K. Mamiya, T. Saitoh, A. Fujimori, K. Ono, A. Kakizaki, T. Ishii, M. Shirai, and T. Kamimura, Phys. Rev. B 57, 8845 (1998).

${ }^{4}$ K. Kusaba, Y. Syono, T. Kikegawa, and O. Shimomura, J. Phys. Chem. Solids 59, 945 (1998).

${ }^{5}$ Y. Fei, C. T. Prewitt, H.-K. Mao, and C. Bertka, Science 268, 1892 (1995).

${ }^{6}$ K. Kusaba, Y. Syono, T. Kikegawa, and O. Shimomura, J. Phys. Chem. Solids 58, 241 (1997).

${ }^{7}$ H. King, D. Virgo, and H. K. Mao, Carnegie Inst. Wash. Publ. 77, 830 (1978).

${ }^{8}$ H. Kobayashi, M. Sato, T. Kamimura, M. Sakai, H. Onodera, N. Kuoda, and Y. Yamaguchi, J. Phys.: Condens. Matter 9, 515 (1997).
${ }^{9}$ S. Minomura and H. G. Drickamer, J. Appl. Phys. 34, 3043 (1963).

${ }^{10}$ C. Karumakaran, V. Vijayakumar, S. Vaidya, S. N. Kunte, and S. Suryanarayana, Mater. Res. Bull. 15, 201 (1980).

${ }^{11}$ G. K. Rozenberg, M. P. Pasternak, G. R. Hearne, and C. C. McCammon, Phys. Chem. Miner. 24, 569 (1997).

${ }^{12}$ G. R. Hearne, M. P. Pasternak, and R. D. Taylor, Rev. Sci. Instrum. 65, 3787 (1994).

${ }^{13}$ R. L. Reichlin, Rev. Sci. Instrum. 54, 1674 (1983).

${ }^{14}$ R. G. Burns, Mineralogical Applications of Crystal Field Theory (Cambridge University Press, Cambridge, England, 1993).

${ }^{15}$ J. B. Goodenough, J. Solid State Chem. 5, 144 (1972).

${ }^{16}$ S. Satpathy, Z. S. Popovic, and F. R. Vukajlovic, Phys. Rev. Lett. 76, 960 (1996).

${ }^{17}$ J. B. Goodenough, Mater. Res. Bull. 13, 1305 (1978).

${ }^{18}$ R. J. Nelmes, M. I. McMahon, S. A. Belmonte, and J. B. Parise, Phys. Rev. B 59, 9048 (1999). 